\title{
ON MAXIMAL SUBGROUPS \\ OF IDEMPOTENT-GENERATED SEMIGROUPS ASSOCIATED WITH BIORDERED SETS
}

\author{
SEAN GARDINER $(D$
}

(Received 19 November 2021; first published online 5 January 2022)

2020 Mathematics subject classification: primary 06A99; secondary 20M10, 20M20.

Keywords and phrases: free idempotent-generated semigroups, biordered sets, maximal subgroups.

It is a long-standing theme of great interest to algebraists, and semigroup theorists in particular, to understand the role that idempotents play in determining the structure or behaviour of an algebra. Howie [6] proved that every semigroup can be embedded in a semigroup generated by idempotents, and Munn [7, 8] discovered a way of recovering information about inverse semigroups from the partial symmetries of their semilattices of idempotents. Nambooripad [9, 10] characterised systems of idempotents of regular semigroups through his invention of biordered sets.

Given any biordered set $E$, we may form the idempotent-generated semigroup $F_{E}$, which is generated by the set $E$, subject to the relations $e f=e * f$ whenever $e$ and $f$ are elements of $E$ and $e * f$ is a basic product. Easdown [4] proved in 1985 that the biordered set of $F_{E}$ is biorder isomorphic to $E$, thus demonstrating that the biordered set axioms, introduced by Nambooripad, characterise certain partial algebras of idempotents of semigroups.

Relatively little is known about the general structure of $F_{E}$, though it is known that every group can arise as a maximal subgroup of $F_{E}$ for some $E$, and that, as a consequence, the word problem is unsolvable (Gray and Ruskuc [5]). We provide a presentation of the maximal subgroups associated with a given $\mathcal{D}$-class of $F_{E}$, which is based on establishing relators that arise from singular squares that are located throughout the graph $\Gamma$ of the $\mathcal{D}$-class. We further apply this machinery to several classes of small examples.

The general difficulty, or intractability, of working with $F_{E}$ is illustrated by the fact that the first example of a biordered set $E$ for which it could be proved that the maximal subgroups are not free did not appear in the literature until 2009, when Brittenham

Thesis submitted to the University of Sydney in February 2021, degree approved on 22 June 2021, supervisor David Easdown.

(C) The Author(s), 2022. Published by Cambridge University Press on behalf of Australian Mathematical Publishing Association Inc. 
et al. [1] exhibited a semigroup with 72 elements with biordered set $E$, such that $F_{E}$ contains maximal subgroups that are free abelian of rank two. In fact, McElwee (private communication) had discovered, but not published, an example prior to this, with 16 elements and maximal subgroups that are cyclic of order two. Dolinka [3] subsequently found a semigroup, in fact a band with 20 elements, also with maximal subgroups that are free abelian of rank two (which is also reconstructed and explored later in this thesis).

The thesis is organised as follows. We begin with a chapter of preliminaries, establishing some notation and terminology, not all of which is standard, and include a method of constructing an ideal extension of a Rees matrix semigroup by semigroup, using transformation and dual transformation semigroups and semigroups of strictly row and column monomial matrices. This construction is a special case of a more general result of Clifford [2], in which he constructs all ideal extensions of completely simple semigroups. The first chapter also gives a thorough introduction to the arrow notation for biordered sets, using Easdown's reformulation of the biordered set axioms. We also explain carefully the definitions related to singular squares in a biordered set $E$ and define $F_{E}$, the semigroup freely generated by elements of $E$, subject to relations arising from basic products in $E$, which occur due to the existence of arrows between elements. The first chapter also provides some foundations for the machinery used to process group presentations, such as Tietze transformations, and fundamental groups of graphs, using, as generators, edges that do not appear in a spanning tree for the graph. We explain how graphs naturally arise from $\mathcal{D}$-classes of a biordered set $E$, which are the connected components of $E$ using paths consisting of alternating double arrows.

The second chapter provides a presentation of the maximal subgroups (all of which are isomorphic) associated with a given $\mathcal{D}$-class of $F_{E}$, which is based on writing down relators that arise from singular squares that can be located throughout the graph $\Gamma$ of the $\mathcal{D}$-class. The technique relies on first choosing a spanning tree for $\Gamma$, then locating directed edges that do not lie on the spanning tree, to be used as generators in the presentation, and then writing down transversals of singular squares in $\Gamma$ to be used as relators.

The third chapter illustrates and applies the technique of the second chapter to find examples of presentations of maximal subgroups associated with certain $\mathcal{D}$-classes of $F_{E}$ in some special cases. One of these is an unpublished example, discovered by McElwee, with maximal subgroups that are cyclic of order two. The underlying semigroup is a band with 16 elements. The second example is due to Dolinka [3], with maximal subgroups that are free abelian of rank two, and the underlying semigroup is again a band, but with 20 elements. In both of these examples, we describe $F_{E}$ fully, up to isomorphism, using the ideal extension theory developed in the first chapter. A third example is given, using the $\mathcal{D}$-class of rank-three idempotents in the full transformation semigroup on five letters, which produces maximal subgroups in $F_{E}$ that are isomorphic to the smallest nonabelian group, namely, the symmetry group of the triangle. 
In the fourth chapter, we show that if $\Gamma$ is isomorphic to a $4 \times 3$ rectangle, then the only possibilities for maximal subgroups are free groups or cyclic groups of order two. We also prove that if the graph $\Gamma$ associated with a $\mathcal{D}$-class has fewer than 12 elements, then all of the associated maximal subgroups of $F_{E}$ are free. In the appendix, we provide a comprehensive list of all graphs that can arise with 12 vertices, which could become the focus of further investigations using the techniques established in this thesis.

\section{References}

[1] M. Brittenham, S. W. Margolis and J. Meakin, 'Subgroups of free idempotent generated semigroups need not be free', J. Algebra 321 (2009), 3026-3042.

[2] A. H. Clifford, 'Extensions of semigroups', Trans. Amer. Math. Soc. 68 (1950), 165-173.

[3] I. Dolinka, 'A note on maximal subgroups of free idempotent generated semigroups over bands', Period. Math. Hungar. 65 (2012), 97-105.

[4] D. Easdown, 'Biordered sets come from semigroups', J. Algebra 96 (1985), 581-591.

[5] R. Gray and N. Ruskuc, 'On maximal subgroups of free idempotent generated semigroups', Israel J. Math. 189 (2012), 147-176.

[6] J. M. Howie, 'The subsemigroup generated by the idempotents of a full transformation semigroup', J. Lond. Math. Soc. (2) 41 (1966), 707-716.

[7] W. D. Munn, 'Uniform semilattices and bisimple inverse semigroups', Q. J. Math. 17 (1966), 151-159.

[8] W. D. Munn, 'Fundamental inverse semigroups', Q. J. Math. 21 (1970), 157-170.

[9] K. S. S. Nambooripad, 'Structure of regular semigroups. I. Fundamental regular semigroups', Semigroup Forum 9 (1975), 354-363.

[10] K. S. S. Nambooripad, 'Structure of regular semigroups. I', Mem. Amer. Math. Soc. 22(224) (1979), 119 pages.

SEAN GARDINER, School of Mathematics and Statistics,

University of New South Wales, Kensington, New South Wales 2052, Australia

e-mail: sean.gardiner@unsw.edu.au 